\title{
Les discours directs libres dans la prose narrative de Stendhal
}

\author{
Christelle Reggiani \\ Université Charles de Gaulle-Lille III \\ christelle.reggiani@gmail.com
}

La solitude du palais épiscopal lui donnait froid. Je puis m'asseoir sur un fauteuil, et ce fauteuil me saisir les bras, j'aurai disparu. À qui ma femme de chambre pourra-t-elle me demander ${ }^{1}$ ?

Des énoncés de ce type, où l'expression de la pensée d'un personnage surgit immédiatement (« librement ») dans un contexte narratif, sont nombreux dans les récits de Stendhal, en particulier dans Le Rouge et le Noir ${ }^{2}$. Un lecteur d'aujourd'hui y reconnaît des occurrences de « discours direct libre », parfaitement conformes à la définition qu'en donne l'ouvrage de référence de Laurence Rosier : « Le discours direct est libre parce qu'il n'est pas introduit pas un verbe, ni marqué typographiquement, mais il est contextuellement signalé » (Rosier 1999 : 295-296).

Or, si l'on suppose ce lecteur quelque peu averti de l'histoire des formes discursives - en l'occurrence énonciatives - un tel constat est pour le moins surprenant, l'usage du discours direct libre restant, dans la langue littéraire, sporadique avant le $\mathrm{XX}^{\mathrm{e}}$ siècle. Il peut, alors, sembler tentant de rapprocher cette liberté discursive de la facture de certains romans contemporains, formellement identique, pour faire de ce phénomène la manifestation énonciative de la modernité d'écriture généralement reconnue à Stendhal (Prévost 1951 [1996] : 91) - modernité en l'occurrence superlative puisqu'elle reconduirait à la figure mystérieuse du précurseur.

C'est contre une telle intuition, et la fausse séduction qu'elle peut exercer, que l'on voudrait s'élever dans les pages qui suivent. On se propose plus précisément, après avoir rappelé brièvement l'histoire des usages littéraires du discours direct libre, d'en examiner précisément l'économie stendhalienne - en se limitant aux deux grands romans achevés, qui mettent au premier plan la vie intérieure des personnages - pour esquisser, in fine, quelques réflexions théoriques sur le type d'historicité qu'engage l'histoire de la langue.

\section{Repères historiques}

Dans sa définition usuelle, le discours direct libre se caractérise à la fois par son absence d'autonomie structurelle, puisqu'il est inséré dans un contexte narratif, et par une indépendance syntaxique (il n'est pas introduit par un verbe de parole ou de pensée) non marquée dans la typographie. Il se distingue donc du monologue autonome aussi bien que du discours direct, "citation-monstration » (Authier-Revuz 1992 et 1993) non régie dont l'autonomie syntaxique est compensée par un signalement typographique précis mobilisant un ensemble de ponctuants (deux points, tiret, guillemets) le cas échéant cumulés (Arabyan et Cunha 2004) - qui, en isolant visuellement la discontinuité énonciative, l'identifie comme telle et la rattache, ce faisant, à son contexte sémantique et pragmatique. C'est dire aussi que le développement du discours direct libre va de pair avec la contestation des incises par la langue littéraire moderne : par la tendance à leur suppression, tout au long du XIX ${ }^{\mathrm{e}}$ siècle, puis leur élargissement au-delà de l'ensemble des verbes de parole, au XX ${ }^{\text {e }}$ siècle (Mylne 1994 : 39-42; Rosier 2008 : 66-67).

Comme souvent, la forme est attestée bien avant sa reconnaissance par les linguistiques, en l'occurrence très tardive puisqu'elle n'intervient pas avant la seconde moitié du XX⿳⺈冂大 siècle (Rosier 1999 : 266-269). 
Plus précisément, on rencontre quelques occurrences de discours directs libres dans la littérature classique, notamment dramatique, où la forme apparaît comme une façon commode - par son économie de moyens pourtant dénuée de la moindre ambiguïté - de rapporter, au sein d'une réplique, des propos qui ne sauraient être assumés, au moment considéré, par le personnage. Le rapport direct de la parole marque ainsi une hétérogénéité minimale, où la discontinuité signale la distance que le locuteur entretient avec un énoncé qui lui appartient cependant; il peut alors s'agir de propos tenus dans un passé plus ou moins lointain, comme dans cette réplique d'Aman (Esther, acte II, scène 1) - où, d'ailleurs, l'identification du discours rapporté n'est pas totalement absente, mais rétrospective (et tardive) :

Je les peignis puissants, riches, séditieux,

Leur dieu même ennemi de tous les autres dieux.

Jusqu'à quand souffre-t-on que ce peuple respire,

Et d'un culte profane infecte votre Empire?

$[\ldots]$

Prévenez, punissez leurs insolents efforts ;

De leur dépouille enfin grossissez vos trésors.

Je dis, et l'on me crut.

Il peut s'agir aussi d'énoncés supposés, en particulier sous la forme de l'écho diaphonique (Roulet 1985 : 71); le théâtre de Marivaux en fournit quelques exemples. Ainsi dans Le Jeu de l'amour et du hasard (acte III, scène 8) :

DORANTE : Vous êtes sensible à son amour, je l'ai vu par l'extrême envie que vous aviez tantôt que je m'en allasse ; ainsi, vous ne sauriez m'aimer.

SILVIA : Je suis sensible à son amour ! qui est-ce qui vous l'a dit? Je ne saurais vous aimer ! qu'en savez-vous? Vous décidez bien vite ${ }^{3}$.

Le discours direct libre reste d'usage sporadique jusqu'à la fin du XIX ${ }^{\mathrm{e}}$ siècle - dans un contexte éditorial en revanche peu favorable, puisqu'il est caractérisé, depuis les années 1850, par une spécialisation et une progression remarquables de l'usage des signes typographiques, notamment pour marquer comme telles les distinctions énonciatives. En voici, cependant, une occurrence balzacienne :

Il aimait, il était jeune, il connaissait Paris ; et sa perspicacité ne lui permettait pas d'ignorer tout ce qu'il y avait d'infamie possible pour une femme élégante, riche, jeune et jolie, à se promener là, d'un pied criminellement furtif. Elle, dans cette crotte, à cette heure ! L'amour que ce jeune homme avait pour cette femme pourra sembler bien romanesque, et d'autant plus même qu'il était officier dans la garde royale ${ }^{4}$.

Même en l'absence de forme verbale conjuguée à un mode personnel, l'emphase graphique de l'italique, associée à la ponctuation exclamative, suggère un soulignement prosodique invitant à une lecture directe de la séquence - dont la validité est du reste confirmée, a posteriori, par la valeur résomptive que porte, en contexte, la proposition suivante, et en particulier le prédicat de « romanesque » attribué à la passion de Maulincour (que justifie, précisément, l'exclamation averbale).

L'usage d'un discours direct sinon libre, du moins non régi, connaît ensuite un regain de faveur dans L'Éducation sentimentale, où des occurrences de discours direct simplement encadrées de guillemets apparaissent régulièrement : 
L'espèce de sous-entendu, d'aveu qu'elle lui avait fait, n'était donc qu'une manœuvre exécutée par intérêt? «Se sont-ils joués de moi ? est-elle complice ? " Une sorte de pudeur, malgré son envie, l'empêchait de retourner chez eux ${ }^{5}$.

Ce type de séquence peut d'ailleurs prolonger, au sein d'un même énoncé, une autre modalité du discours rapporté ; ainsi dans Salammbô : « [...] le vieux pilote baissa la voix pour annoncer qu'une trirème était prise à Rusicada par les Numides, - "car ils sont avec eux, Maître"6"».

Mais on trouve aussi dans L'Éducation sentimentale une demi-douzaine d'occurrences dépourvues de toute marque graphique :

Frédéric souffrait des nerfs. Deslauriers n'en crut rien. Devant une pareille douleur, il avait senti se réveiller sa tendresse, et il le réconforta. Un homme comme lui se laisser abattre, quelle sottise ! Passe encore dans la jeunesse, mais plus tard, c'est perdre son temps ${ }^{7}$.

[Sénécal] commença par demander du pain de ménage (le plus ferme possible), et, à ce propos, parla des meurtres de Buzançais et de la crise des subsistances.

Rien de tout cela ne serait survenu si on protégeait mieux l'agriculture, si tout n'était pas livré à la concurrence, à l'anarchie, à la déplorable maxime du « laissez faire, laissez passer »! Voilà comment se constituait la féodalité de l'argent, pire que l'autre ! Mais qu'on y prenne garde ! le peuple, à la fin, se lassera, et pourrait faire payer ses souffrances aux détenteurs du capital, soit par de sanglantes proscriptions, ou par le pillage de leurs hôtels ${ }^{8}$.

Malgré l'absence de pronoms de la première personne, les marques temporelles («c'est perdre son temps », «le peuple se lassera ») interdisent une interprétation indirecte de ces séquences. D'autres occurrences demeurent, en revanche, d'interprétation ambiguë, par défaut de marquage personnel ou temporel dans des contextes où sont présentes d'autres modalités du discours rapporté : «Frédéric ne pouvait distinguer sa figure. Un soupçon le saisit, il lui sembla que c'était Mme Arnoux. Impossible, cependant ! Pourquoi seraitelle venue ${ }^{9}$ ? » De même, dans Bouvard et Pécuchet :

[...] Bouvard jeta son havresac par terre, en déclarant qu'il n'irait pas plus loin. La géologie est trop défectueuse ! À peine connaissons-nous quelques endroits de l'Europe. Quant au reste, avec le fond des Océans, on l'ignorera toujours. Enfin, Pécuchet ayant prononcé le mot de règne minéral : — « Je n'y crois pas, au règne minéral ! ${ }^{10}$

La forme est ensuite reprise par Zola, dont les romans présentent des occurrences nombreuses, quoique ponctuelles, de discours directs libres d'interprétation presque toujours incertaine, dans la mesure où le caractère généralisant de l'énoncé (comme dans Bouvard et Pécuchet) permet son attribution au narrateur aussi bien qu'à tel personnage, «amortissant» ainsi la rupture énonciative - comme dans ce passage où l'empathie du narrateur se confond avec la compassion de Gervaise :

Alors, le pauvre petit corps de la mourante apparut. Ah! Seigneur ! quelle misère et quelle pitié ! Les pierres auraient pleuré. Lalie était toute nue, un reste de camisole aux épaules en guise de chemise ; oui, toute nue, et d'une nudité saignante et douloureuse de martyre. Elle n'avait plus de chair, les os trouaient la peau. [...] Des pieds à la tête, elle n'était qu'un noir. Oh! ce massacre de l'enfance, ces lourdes pattes d'homme écrasant cet amour de quiqui, cette abomination de tant de faiblesse râlant sous une pareille croix ! On adore dans les églises des saintes fouettées dont la nudité est moins pure. Gervaise, de nouveau, s'était accroupie, ne songeant plus à tirer le drap, renversée par la vue de ce rien du tout pitoyable, aplati au fond du lit; et ses lèvres tremblantes cherchaient des prières ${ }^{11}$.

Intervient alors, pour une part, la tendance de la seconde moitié du XIX ${ }^{\mathrm{e}}$ siècle à réserver les guillemets au marquage de la pensée, ou de la parole murmurée, les répliques à haute voix n'étant plus signalées que par des tirets (les guillemets disparaissent d'abord des dialogues, puis des répliques isolées [Arabyan et Cunha 2004 : 42]), sans que cette division du travail typographique entre pensée et parole tout à fait oralisée puisse être le seul facteur d'explication en cause. 
Le discours direct libre connaît ensuite une éclipse relative, pour revenir massivement dans le cadre de la véritable expérimentation énonciative collective qui définit, pour une part, l'écriture romanesque des années trente - un retour qui tient sans doute à la diffusion du "patron » stylistique de l'écriture de la pensée, d'autant plus forte qu'elle est portée par la forme narrative du monologue intérieur (autonome) dans l'entredeux-guerres (Philippe 2009a), puisque l'insertion immédiate de paroles ou de pensées dans un contexte narratif (le plus souvent constitué, du reste, par les autres modalités du discours rapporté) semble dès lors formellement acceptable - autrement dit lisible.

La « liberté » du discours rapporté caractérise, notamment, la facture, en effet très mobile du point de vue énonciatif, du roman aragonien :

Il se débattait pourtant contre la certitude qu'il ne se rendormirait pas. La certitude de cette présence écartée, mais implacable, contre cette peur qui se forme dans les ténèbres. Non, je ne penserai pas à l'argent. Et puis cela s'installait avec ce souverain mépris de leur victime qu'ont les rêves éveillés. Cela lui séchait la gorge, lui fouaillait le ventre. Il cherchait à se perdre dans des images suscitées, des préoccupations rappelées avec panique. Quelle série noire à la Bourse ! Il faut absolument penser à autre chose. Le diorama nocturne de la petite ville, du lycée, des fantoches de la vie de province, fournissait de faibles diversions, de pâles silhouettes à cette angoisse où finalement Mercadier retombait ${ }^{12}$.

Dans la seconde moitié du $\mathrm{XX}^{\mathrm{e}}$ siècle, le discours direct libre apparait comme une ressource usuelle de l'écriture narrative. En témoignent, notamment (entre bien d'autres exemples possibles), la manière de Marguerite Duras - «Il dit qu'il est chinois, que sa famille vient de la Chine du Nord, de Fou-Chouen. Voulez-vous me permettre de vous ramener chez vous à Saïgon? Elle est d'accord ${ }^{13} »-$ ou celle de Jean Échenoz - « [...] lorsqu'il ferme un instant les yeux, il ressemble à son masque mortuaire. Vous n'êtes pas bien? Il dit que ça va, que ça devrait aller mais qu'il se sent encore très fatigué ${ }^{14} \gg-$ jusqu'à la prose du $\mathrm{XXI}^{\mathrm{e}}$ siècle, que représenteront ici deux exemples empruntés respectivement (et chronologiquement) à des œuvres de Christine Montalbetti et de Laurent Mauvignier :

\begin{abstract}
Jacques pose sa redingote sur la chaise, elle y semble une immense chauve-souris fatiguée, inutile de la suspendre correctement quand dans moins d'une heure, Qu'est-ce que je dis, ça me fait à peine trente minutes, il lui faudra la réendosser, valise à la main cette fois, direction l'arrêt de la diligence de terre ${ }^{15}$.
\end{abstract}

Mais Bernard regarde une dernière fois dans l'entrée du bar où hélas personne ne vient - cette idée que personne ne vienne, il regarde sa montre, est-ce que vraiment personne ne va venir ${ }^{16}$ ?

\title{
2 L'économie typographique de la prose stendhalienne
}

Cette histoire une fois retracée à grands traits ${ }^{17}$, on s'attardera sur les pratiques énonciatives de Stendhal, dont la relative singularité sollicite l'attention - en commençant donc par décrire, de façon générale, l'économie discursive de ses récits.

S'agissant de la parole oralisée, elle y est couramment surmarquée, dans la mesure où la présence d'un verbe (ou d'une lexie) de parole s'accompagne d'un ensemble (aux contours mobiles) de divers indices typographiques : balises visuelles de l'alinéa, du tiret et des deux-points, plus rarement des guillemets ${ }^{18}$, voire de l'italique ${ }^{19}$, dont Stendhal tend cependant à réserver l'hétérogénéité graphique au marquage de l'altérité impersonnalité du on-dit, énonçant les lieux communs contre lesquels s'enlève la particularité des discours pleinement subjectifs, ou étrangeté d'une autre langue, d'un autre texte (Dürrenmatt 1998: 57-67 et 2003 : 358). Quant à la pensée, son signalement textuel combine, selon des actualisations variées, la présence de verbes (ou de lexies) spécifiques, le cas échéant sous la forme d'incises, à une économie typographique particulière : si les deux-points sont souvent présents lorsque le verbe de pensée ne constitue pas le noyau d'une incise, de même que le retrait de l'alinéa, l'usage des guillemets est, en revanche, relativement rare, aucune de ces marques n'étant d'ailleurs requise par la manière stendhalienne. On aboutit ainsi à des discours 
rapportés absolument « libres », au sens où ils ne sont ni régis, ni graphiquement marqués : « Mme de Rênal tomba sur une chaise, presque évanouie de douleur. Il va humilier Julien, et par ma faute ! Elle eut horreur de son mari, et se cacha la figure avec les mains » $(R N, 385)$. Singulière économie, donc, que cette facture énonciative, aux deux sens que peut connaître le terme, puisque le système qui commande la mise en page des discours (oralisés et intérieurs) repose sur la raréfaction du signalement typographique.

Si de tels discours directs « libres »- exclusivement réservés à 1'expression de la pensée ${ }^{20}$ - ne suscitent « nulle obscurité ni trouble », comme le remarque récemment le philosophe Jean-Louis Chrétien (2009: 62), c'est qu'on a, en fait, affaire à un continuum, où le degré le plus faible du marquage ne constitue jamais, parce qu'il intervient dans un certain contexte, un «degré zéro » (l'exemple ci-dessus le montre suffisamment) (Rosier 1999 : 296).

Si l'on considère les combinaisons possibles des deux variables que sont l'attribution de la citation et son signalement graphique, on obtient les quatre configurations suivantes, définissant les quatre principaux degrés de l'échelle : le discours direct peut être explicitement attribué par son contexte immédiat en même temps que graphiquement signalé ; il peut être attribué (en contexte immédiat) sans être graphiquement signalé, graphiquement marqué sans être explicitement attribué ou, enfin, n'être ni attribué ni graphiquement marqué.

Ces différents degrés sont tous les quatre présents dans la prose de Stendhal - même si l'absence de marques qui caractérise le quatrième en rend la forme paradoxalement plus notable.

\subsection{Attribution et marquage graphique}

Si la prose stendhalienne ne fait pas preuve d'une inventivité particulière en ce qui concerne les lexèmes attributifs, elle les associe, en revanche, à une diversité remarquable de signaux graphiques : deux-points et majuscule sont possibles - «Sa pensée ne s'arrêta pas davantage à ce raisonnement. [...] Le moment de la mort ne l'arrêtait guère plus : J'y songerai après le jugement » $(R N, 759)$ - mais également la balise minimale que constitue le décrochement de l'alinéa :

Serait-ce la force du sang? se dit l'abbé à demi-voix et comme se parlant à soi-même. « Ce qu'il y a de singulier, ajouta-t-il en regardant Julien, c'est que le marquis vous connaît... » $(R N, 560)$.

Quant aux guillemets, ils sont associés de manière privilégiée à des propos donnés comme écrits (lettres, billets...) - ainsi dans ce passage de La Chartreuse de Parme où le murmure de la voix tout juste audible redouble l' « impression » privée d'un sonnet :

Il s'approcha d'elle et prononça, à demi-voix et comme se parlant à soi-même, deux vers de ce sonnet de Pétrarque, qu'il lui avait envoyé du lac Majeur, imprimé sur un mouchoir de soie : «Quel n'était pas mon bonheur quand le vulgaire me croyait malheureux, et maintenant que mon sort est changé !» $(C P, 479)$.

\subsection{Attribution sans marquage graphique}

Le plus souvent, en particulier dans La Chartreuse de Parme, où l'usage des deux-points est pour le moins parcimonieux, un verbe de parole ou de pensée («parler» et «considérer», dans les exemples suivants) précède immédiatement la séquence de discours rapporté :

Le comte Mosca, interrogé, parla de sa vie à Parme. En Espagne, sous le général Saint-Cyr, j'affrontais des coups de fusil pour arriver à la croix et ensuite à un peu de gloire, maintenant je m'habille comme un personnage de comédie pour gagner un grand état de maison et quelques milliers de francs $(C P$, 123). 


\begin{abstract}
Il n'en trouvait plus d'aussi bonnes [raisons] quand il venait à considérer son âge et les soucis quelquefois fort tristes qui remplissaient sa vie. Un homme habile à qui la peur ôte l'esprit me donne une grande existence et beaucoup d'argent pour être son ministre ; mais que demain il me renvoie, je reste vieux et pauvre, c'est-à-dire tout ce qu'il y a au monde de plus méprisé ; voilà un aimable personnage à offrir à la comtesse ! $(C P, 127)$.
\end{abstract}

Le lexème attributif peut être aussi bien constitué par un substantif désignant une « faculté de l'âme » - ainsi $\mathrm{du}$ « cœur » (dans une version quelque peu hétérodoxe de la philosophie du temps) : « Julien l'embrassa, mais à l'instant la main de fer du devoir saisit son cœur. Si elle voit combien je l'adore, je la perds. Et, avant de quitter ses bras, il avait repris toute la dignité qui convient à un homme » $(R N, 732)$ - voire le résultat de cette activité de l'esprit, disposition mentale ou affect : "Il avait des remords. Dans quelle situation est-ce que je mets ce pauvre comte Mosca, lui ministre de la police !» $(C P, 247)$.

\title{
2.3 Marquage graphique sans attribution explicite
}

Les deux-points, associés à la majuscule et aux guillemets, signalent alors visuellement l'ouverture d'une séquence de discours rapporté : « À 6 heures le marquis le fit demander, il regarda avec une peine évidente les bottes de Julien : "J'ai un tort à me reprocher, je ne vous ai pas dit que tous les jours à 5 heures et demie, il faut vous habiller" » $(R N, 568)$.

\subsection{Discours directs « libres » (sans attribution ni marquage graphique)}

Il faut ici distinguer les exemples de ce type - « Il prit un grand détour. Si ce diable d'abbé Castanède m'a reconnu, il n'est pas homme à perdre facilement ma trace » $(R N, 700)$ - de ceux où le signalement du discours rapporté est simplement décalé.

Il peut alors s'agir d'anticipation, notamment dans le cas (fréquent) de deux séquences disjointes de discours direct dont seule la première est signalée comme telle :

Tout en écoutant les récits un peu longs de monseigneur Landriani, il se disait : Aurais-je dû tirer un coup de pistolet au valet de chambre qui tenait par la bride le cheval maigre ? Sa raison lui disait oui, mais son cœur ne pouvait s'accoutumer à l'image sanglante du beau jeune homme tombant de cheval défiguré.

Cette prison où j'allais m'engloutir, si le cheval eût bronché, était-elle la prison dont je suis menacé par tant de présages?

Cette question était de la dernière importance pour lui, et l'archevêque fut content de son air de profonde attention $(C P, 199)$.

Mais la situation la plus courante est celle du marquage rétrospectif (pour autant parfaitement clair) - soit qu'une incise se trouve insérée dans la deuxième phrase d'une séquence de discours direct :

Depuis quelques instants Fabrice s'était remis en marche; il passa la colline qui forme la presqu'île de Durini, et enfin parut à ses yeux ce clocher du village de Grianta, où si souvent il avait fait des observations d'étoiles avec l'abbé Blanès. Quelle n'était pas mon ignorance en ce temps-là ! Je ne pouvais comprendre, se disait-il, même le latin ridicule de ces traités d'astrologie que feuilletait mon maître $[\ldots](C P, 177)$.

- soit qu'un substantif (« réflexion», dans l'exemple suivant) vienne catégoriser a posteriori l'activité mentale précédemment rapportée, la signalant ainsi explicitement comme telle :

Les longues crinières pendantes que portaient à leurs casques les dragons de la suite l'empêchèrent de distinguer les figures. Ainsi, je n'ai pu voir l'Empereur sur un champ de bataille, à cause de ces maudits verres d'eau-de-vie ! Cette réflexion le réveilla tout à fait $(C P, 79)$. 
Si la présence de ce type de discours rapportés n'est jamais problématique du point de vue de l'interprétation, son insertion connaît cependant les deux configurations inverses de l' "amortissement " (où une "phrase tampon » au discours indirect, le cas échéant libre, constitue une transition formelle vers le discours direct « libre ») et de la solution de continuité la plus radicale, lorsque le décrochement énonciatif intervient dans les limites d'une seule et même phrase.

On citera d'abord des exemples de la première situation, mobilisant le discours indirect, puis le discours indirect libre :

Quand Julien aperçut les ruines pittoresques de l'ancienne église de Vergy, il remarqua que depuis l'avant-veille il n'avait pas pensé une seule fois à Mme de Rênal. L'autre jour en partant, cette femme m'a rappelé la distance infinie qui nous sépare, elle m'a traité comme le fils d'un ouvrier. Sans doute elle a voulu me marquer son repentir de m'avoir laissé sa main la veille... $(R N, 417)$.

Le prince se mit en colère; il voyait tout son bonheur lui échapper. Que devenir après que la duchesse aurait quitté sa cour? D'ailleurs, quelle humiliation d'être refusé ! Enfin qu'est-ce que va me dire mon valet de chambre français quand je lui conterai ma défaite ? $(C P, 482)$.

Le discours direct (ici graphiquement marqué par l'alinéa) peut se trouver ainsi encadré de séquences de discours indirect libre :

La profondeur, l'inconnu du caractère de Julien, eussent effrayé, même en nouant avec lui une relation ordinaire. Et elle allait en faire son amant, peut-être son maitre !

Quelles ne seront pas ses prétentions, si jamais il peut tout sur moi ? Eh bien! je me dirai comme Médée : Au milieu de tant de périls, il me reste MoI.

Julien n'avait nulle vénération pour la noblesse du sang, croyait-elle. Bien plus, peut-être il n'avait nul amour pour elle ! $(R N, 645)$

Quant à la deuxième configuration, ses occurrences sont très rares : au nombre de deux (à ma connaissance) dans Le Rouge et le Noir, on en rencontre une demi-douzaine dans La Chartreuse de Parme; presque toujours, c'est le point-virgule qui signale le changement de plan énonciatif. On citera donc exhaustivement les occurrences du Rouge (au motif de leur rareté) - d'ailleurs d'orientations inverses, du discours vers le récit, puis du récit vers le discours - pour renvoyer en note les exemples trouvés dans La Chartreuse :

Julien resta profondément humilié du malheur de n'avoir su que répondre à Mme de Rênal.

Un homme comme moi se doit de réparer cet échec, et saisissant le moment où l'on passait d'une pièce à l'autre, il crut de son devoir de donner un baiser à Mme de Rênal $(R N, 422)$.

À force d'examiner le comte Norbert, Julien remarqua qu'il était en bottes et en éperons ; et moi je dois être en souliers, apparemment comme inférieur. On se mit à table $(R N, 569)^{21}$.

Dans tous les cas, on constate que l'absence d'attribution explicite dans le contexte immédiat ne signifie pas une «liberté » absolue du discours rapporté : l'examen de ce contexte, antérieur et/ou postérieur, le cas échéant élargi, permet de vérifier qu'un certain nombre d'éléments préparent (ou désignent a posteriori) l'apparition de la pensée citée. Au fond, un discours rapporté ne saurait être, à proprement parler, absolument " libre », sauf à occulter, précisément, le rapport qui le constitue comme tel. C'est dire que les discours directs libres sont toujours, d'une certaine façon, «progressive[ment] insér[és] (Rosier 1999 : 295), cette relation au contexte prenant, le cas échéant, la forme d'une double obliquité, combinant indirection sémantique (l'idée de pensée est connotée, non dénotée, par le contexte [voir Rosier 1999: 282]) et distance textuelle (l'attribution peut être déplacée par rapport au discours cité, qu'il s'agisse d'une anticipation ou d'un retard, éventuellement conjugués). 


\section{Des discours directs libres?}

Malgré une apparente identité formelle, il semble donc préférable de renoncer, s'agissant de la prose de Stendhal, à la dénomination anachronique de « discours direct libre » : les discours rapportés que nous venons de citer ne sauraient évidemment se libérer d'une norme typographique qui leur est postérieure ${ }^{22}$, et une dénomination plus neutre - celle, par exemple, de discours directs non régis - est sans doute préférable. C'est pourquoi, plutôt que de faire de Stendhal un improbable précurseur de la souplesse énonciative contemporaine, on préférera voir dans la liberté des discours intérieurs stendhaliens celle que leur confère la sobriété de la mise en page classique (et non la libération d'une norme typographique solidement établie - à la différence de certains romans du $\mathrm{XX}^{\mathrm{e}}$ et $\mathrm{du} \mathrm{XXI}^{\mathrm{e}}$ siècles).

La relative singularité de la facture discursive stendhalienne s'établit ainsi à la conjonction de deux possibles de la langue littéraire du temps, combinant sobriété typographique classique et raréfaction moderne des incises (Mylne 1994: 45 et Rosier 1999: 256) ${ }^{23}$. Cette désinvolture énonciative ${ }^{24}$, qu'autorisent, sans l'imposer, les modes de la prose narrative d'alors, reconduit, à l'évidence, à une motivation esthétique : il est sûr que le goût stendhalien de la brevitas (Crouzet 1981 : 401-403) trouve ainsi une traduction énonciative immédiate, la « liberté » des décrochements discursifs donnant, de ce point de vue linguistique-là, sa forme esthétique la plus appropriée à l'énergie passionnelle d'une spontanéité mentale qui semble d'emblée verbale (voir Prévost 1951 [1996] : 368, puis Cohn 1981: 76 et Lassagne 2007) - ainsi saisie sur le même plan exactement (comme au théâtre) que les actions diverses (événements, paroles...) qui constituent la trame du récit.

De ce parcours stendhalien découle une double leçon de méthode.

Simple leçon de prudence philologique, d'abord, où l'on s'accordera aisément sur l'absurdité historique qu'il y aurait à vouloir saisir un fait de langue abstrait de tout contexte - saisi isolément, un fait de langue est, en tant que tel, dépourvu de sens : pour revenir aux exemples précédents, il est clair qu'une absence de marques typographiques n'a pas la même valeur selon qu'elle actualise un possible de la langue écrite, ou représente une infraction à une norme établie. Dès lors qu'il s'agit d'en faire un objet d'histoire, un fait de langue ne vaut en somme qu'à être considéré au sein d'un système ${ }^{25}$.

S'ensuit aussi une réflexion plus générale, d'ordre épistémologique, sur la " méthode de l'histoire littéraire », comme le disait Lanson. Il est sûr, en effet, que c'est la culture linguistique particulière propre au «moment énonciatif » dans lequel nous sommes (Philippe 2002 : 218) qui nous porte à reconnaître dans la prose de Stendhal des occurrences nombreuses de discours direct libre. Il est, du reste, frappant que le phénomène soit totalement absent des grands livres de Jean Prévost (1951) et de Georges Blin (1954), qui consacrent pourtant l'un et l'autre des développements importants à la question de l'expression de la pensée, ainsi que de la thèse de Josiane Attuel sur Le Style de Stendhal (1980), alors qu'il est signalé (après Booker 1985, puis Crouzet 1995) et commenté dans l'ouvrage, par ailleurs absolument non technique, de Jean-Louis Chrétien sur le monologue intérieur, qui le décrit comme un « passage spontané au monologue intérieur » (Chrétien 2009 : 49 et $\left.62^{26}\right)$.

Si l'on peut alors à bon droit parler d'effet de perspective, il ne s'agit pas, pour autant, d'un défaut de méthode. De fait, le discours historique, parce qu'il est nécessairement rétrospectif - et l'histoire de la langue n'échappe pas, bien sûr, à ce régime général - est faiblement cumulatif : chaque moment de l'histoire doit, pour son propre compte, refaire le parcours de ceux qui l'ont précédé, y trouvant, le cas échéant, les « précurseurs » qui répondent à ses propres préoccupations.

L'écriture de l'histoire propose ainsi de curieuses généalogies, d'orientation inverse : à l'idée d'une téléologie (Philippe 2006 : 171), imaginairement fondée sur le modèle biologique de la filiation, elle substitue une archéologie - en un sens point trop éloigné de celui que Foucault (1969) donnait à ce terme. C'est du reste pourquoi le modèle archéologique (cette fois au sens usuel du terme) de l'invention semble, en l'occurrence, 
parfaitement approprié : de même que la trouvaille archéologique est en fait une redécouverte, mettant au jour ce qui était déjà là (ce qui ne préjuge pas, s'agissant de l'histoire de la langue, de la possibilité de véritables inventions stylistiques - même si l'idée d'un commencement absolu semble, ici comme en toute matière humaine, problématique), les prosateurs $\mathrm{du} \mathrm{XX}^{\mathrm{e}}$ siècle «inventent» une liberté du discours rapporté dont Stendhal, dans un contexte bien différent, avait déjà montré l'exemple. À l'instar des fables et des contes de La Fontaine pour le discours indirect libre, les récits de Stendhal ont ainsi proposé, de manière assez isolée en leur temps, des usages quantitativement, donc qualitativement, nouveaux de formes discursives disponibles avant qu'elles ne tombent ensuite en désuétude, faute de motivations esthétiques, constituant alors une réserve de la langue littéraire.

C'est pourquoi l'on peut légitimement préférer, à l'absurde figure du précurseur, l'idée oulipienne de « plagiat par anticipation » (Bayard 2009), dont l'apparente fantaisie temporelle cerne en fait exactement le mouvement paradoxal de l'histoire des formes, où les possibles de la langue (en l'occurrence littéraire) tendent à n'être reconnus comme tels qu'après-coup : ainsi le goût de Stendhal pour le discours direct libre ne pouvait-il être désigné qu'à la fin $\mathrm{du} \mathrm{XX}^{\mathrm{e}}$ siècle. Curieuse rencontre, du reste, où l'histoire énonciative de la langue du roman donne sa forme proprement discursive à l'extra-temporalité dont la visée fonde, justement, la poétique de l'écrivain : « Je pourrais faire un ouvrage qui ne plairait qu'à moi, qui serait reconnu beau en 2000 » (Journal, 31 décembre 1804, cité par Prévost 1951 [1996] : 91).

\section{Références bibliographiques}

Arabyan, M. et Cunha, D. (2004). «La ponctuation du discours direct des origines à nos jours ». L'Information grammaticale, $\mathrm{n}^{\circ} 102$, p. 35-45.

Attuel, J. (1980). Le Style de Stendhal. Efficacité et romanesque. Bologne-Paris : Pàtron-Nizet.

Authier-Revuz, J. (1992). «Repères dans le champ du discours rapporté ». L'Information grammaticale, $\mathrm{n}^{\circ}$ 55, p. 37-41.

Authier-Revuz, J. (1993). « Repères dans le champ du discours rapporté ». L'Information grammaticale, n 56, p. 9-14.

Bayard, P. (2009). Le Plagiat par anticipation. Paris : Minuit.

Berthier, P. et Bordas, É. (éd.) (2005). Stendhal et le style. Paris : Presses Sorbonne nouvelle.

Blin, G. ([1954] 1990). Stendhal et les problèmes du roman. Paris : José Corti.

Booker, J. T. (1985). « Style direct libre : the Case of Stendhal ». Stanford French Review, n 2, p. 137-151.

Chrétien, J.-L. (2009). Conscience et Roman, I. La Conscience au grand jour. Paris : Minuit.

Cohn, D. (1981). La Transparence intérieure. Modes de représentation de la vie psychique dans le roman, trad. Alain Bony. Paris : Seuil.

Crouzet, M. (1981). Stendhal et le langage. Paris : Gallimard.

Crouzet, M. (1995). Le Rouge et le Noir. Essai sur le romanesque stendhalien. Paris : PUF.

Dürrenmatt, J. (1998). Bien coupé mal cousu. De la ponctuation et de la division du texte romantique. Saint-Denis : Presses Universitaires de Vincennes.

Dürrenmatt, J. (2003). «Italiques ». In Ansel, Y., Berthier, P. et Nerlich, M. (éd.), Dictionnaire de Stendhal, Paris : Honoré Champion, p. 358.

Foucault, M. (1969). L’Archéologie du savoir. Paris : Gallimard.

Lassagne, L. (2007). Ce que se parler veut dire. La Représentation du monologue dans les romans de Stendhal, thèse de doctorat dirigée par Françoise Mélonio. Paris : université Paris IV-Sorbonne. 
Lips, M. (1926). Le Style indirect libre. Paris : Payot.

Mylne, V. G. (1994). Le Dialogue dans le roman français de Sorel à Sarraute. Paris : Universitas.

Philippe, G. (2002). Sujet, verbe, complément. Le moment grammatical de la littérature française (1890-1940). Paris : Gallimard.

Philippe, G. (2006). «Langue littéraire et téléonomie des changements linguistiques (à propos de la "syntaxe impressionniste") ». In Berlan, F. (éd.), Langue littéraire et Changements linguistiques, Paris : Presses de l'Université Paris-Sorbonne, p. 163-171.

Philippe, G. (2009a). «La langue littéraire, le phénomène et la pensée ». In Philippe, G. et Piat, J. (éd.), La Langue littéraire. Une histoire de la prose en France de Gustave Flaubert à Claude Simon, Paris : Fayard, p. 91-119.

Philippe, G. (2009b). « L’invention de la prose ». In Philippe, G. et Piat, J. (éd.), La Langue littéraire, op. cit., p. 323-343.

Philippe, G. (à paraître). «Par faisceaux et par moments : sur la possibilité d'une histoire stylistique de la littérature ». In Gardes-Tamine J. et Molinié G. (éd.), Style et création.

Prévost, J. ([1951] 1996). La Création chez Stendhal. Paris : Gallimard, coll. « Folio Essais ».

Reggiani, C. (2009). «Le texte romanesque : un laboratoire des voix». In Philippe, G. et Piat, J. (éd.), La Langue littéraire, op. cit., p. 121-154.

Rosier, L. (1999). Le Discours rapporté. Histoire, théories, pratiques. Bruxelles : Duculot.

Rosier, L. (2008). Le Discours rapporté en français. Paris : Ophrys.

Roulet, E. (1985). «Structures hiérarchiques et polyphoniques du discours». In Roulet, E. et al., L'Articulation du discours en français contemporain, Berne : Peter Lang, p. 9-83.

Stolz, C. (2001). «Les discours directs libres dans Les Voyageurs de l'impériale, voix hétérogènes du monde réel ». In Fromilhague, C. (éd.), Styles, genres, auteurs, vol. I, Paris : Presses de l’Université Paris-Sorbonne, p. 163-175.

${ }^{1}$ Stendhal, Le Rouge et le Noir (1830), Euvres romanesques complètes, Gallimard, coll. « Bibliothèque de la Pléiade», 2005, p. 766 (les éditeurs précisent que «la ponctuation [de Stendhal] a été conservée» [p. LXXXV]). Les références à ce roman, ainsi qu'à La Chartreuse de Parme (1839) (GF-Flammarion, 1964), seront désormais données au fil du texte entre parenthèses, respectivement précédées de la mention $R N$ ou $C P$ (la ponctuation des occurrences empruntées à $L a$ Chartreuse a été systématiquement vérifiée dans l'exemplaire Chaper).

2 Le Rouge et le Noir en compte une cinquantaine d'occurrences, et La Chartreuse de Parme une vingtaine.

${ }^{3}$ De même, dans Le Legs (scène 23), où le discours supposé est « en substance » (Authier-Revuz 1992: 41) (« en un mot») : «Il a commencé par établir que je ne pouvais pas le souffrir. En un mot, je le déteste, je suis furieuse contre son amour ; voilà d'où il part [...].»

${ }^{4}$ Honoré de Balzac, Ferragus (1833), La Comédie humaine, Gallimard, coll. « Bibliothèque de la Pléiade », t. V, 1977, p. 796-797.

5 Gustave Flaubert, L'Éducation sentimentale (1869), Le Livre de poche, 1983, p. 223. Ce patron typographique ne tombera pas, du reste, en désuétude, et Malraux, par exemple, le reprendra au $\mathrm{XX}^{\mathrm{e}}$ siècle : «Ce pied vivait comme un animal endormi. Terminait-il un corps? "Est-ce que je deviens imbécile ?" Il fallait voir ce corps. Le voir, voir cette tête ; pour cela, entrer dans la lumière, laisser passer sur le lit son ombre trapue. Quelle était la résistance de la chair ? Convulsivement, Tchen enfonça le poignard dans son bras gauche » (André Malraux, La Condition humaine [1933], Gallimard, coll. « Folio », 1984, p. 10).

${ }^{6}$ Gustave Flaubert, Salammbô (1862), GF-Flammarion, 2001, p. 143.

7 L'Éducation sentimentale, op. cit., p. 82. 
${ }^{8}$ Op. cit., p. 163.

${ }^{9}$ Op. cit., p. 240.

${ }^{10}$ Gustave Flaubert, Bouvard et Pécuchet (1880), GF-Flammarion, 1994, p. 159.

11 Émile Zola, L'Assommoir (1877), Le Livre de poche, coll. « Classique », 1996, p. 464.

12 Louis Aragon, Les Voyageurs de l'impériale (1939-1948), Gallimard, coll. « Folio », 1996, p. 337. Voir Stolz (2001).

13 Marguerite Duras, L'Amant, Minuit, 1984, p. 44.

14 Jean Échenoz, Ravel, Minuit, 2006, p. 13.

${ }^{15}$ Christine Montalbetti, L'Origine de l'homme, POL, 2002, p. 24.

${ }^{16}$ Laurent Mauvignier, Des hommes, Minuit, 2009, p. 211.

${ }^{17}$ Ce développement complète, et précise sur certains points, Reggiani 2009 : 140-148.

18 On relève, dans La Chartreuse de Parme, un emploi atypique des guillemets pour signaler une traductionainsi glosée par le narrateur: «Je demande pardon pour cette phrase [placée entre guillemets], traduite de l'italien » $(C P, 126)$.

19 «[...] enfin Fabrice arrive à Ludovic auquel il crie : Tue ! tue tout ce qui porte des torches ! » $(C P, 250)$.

${ }^{20}$ Cet exemple, parmi d'autres, le montre bien : «Elle lui reprocha le soir, en riant, son goût pour Mme de Fervaques : un bourgeois aimer une parvenue ! Les cœurs de cette espèce sont peut-être les seuls que mon Julien ne puisse rendre fous. Elle avait fait de vous un vrai dandy, disait-elle en jouant avec ses cheveux » $(R N, 734)$. Il n'existe, à ma connaissance, qu'une exception à ce principe, dans La Chartreuse de Parme : «Alors il lui expliqua avec beaucoup de pédanterie qu'à l'armée il faut appartenir à un corps et porter un uniforme, faute de quoi il est tout simple qu'on vous prenne pour un espion. L'ennemi nous en lâche beaucoup ; tout le monde trahit dans cette guerre. Les écailles tombèrent des yeux de Fabrice ; il comprit pour la première fois qu'il avait tort dans tout ce qui lui arrivait depuis deux mois » $(C P, 91)$.

21 «Elle se voyait sur le Corso, à Milan, heureuse et gaie comme au temps du vice-roi ; la jeunesse, ou du moins la vie active, recommenceraient pour moi !» $(C P, 131)$; « ̀̀ la vue de tant de prudence le prince eut presque de l'humeur; il paraît que voici un homme d'esprit qui nous arrive de Naples, et je n'aime pas cette engeance; un homme d'esprit a beau marcher dans les meilleurs principes et même de bonne foi, toujours par quelque côté il est cousin germain de Voltaire et de Rousseau » $(C P, 158)$; «Toutes sortes d'idées folles arrivèrent à la tête de notre héros, qui dans ce moment n'était pas fort logique. Par exemple, il songea à s'enfuir par la porte du bureau qui était restée ouverte ; je me défais de mon habit; je me jette dans le Pô, et sans doute je pourrai le traverser à la nage. Tout vaut mieux que le Spielberg » $(C P, 212-213) ;$ " [...] quels que soient mes sentiments envers la duchesse, il ne faut point oublier que c'est une des plus grandes dames de ma cour. Comment Louis XIV parlait-il aux princesses ses filles quand il avait lieu d'en être mécontent? et ses yeux s'arrêtèrent sur le portrait du grand roi » $(C P, 261)$.

22 Comme l'écrit Laurence Rosier : «L'effet d'étrangeté produit par les exemples où il y a une absence totale de marques typographiques s'ancre dans l'habitude du lecteur moderne, qui consiste à juxtaposer deux plans d'énonciation en le marquant par la typographie » (Rosier 1999 : 283).

23 Il faut rappeler ici la présence de quelques occurrences de «discours directs libres » dans L'Émile de Rousseau - déjà relevées par Marguerite Lips : «À ce nom de Sophie, vous eussiez vu tressaillir Émile. Frappé d'un nom si cher, il se réveille en sursaut et jette un regard avide sur celle qui l'ose approcher. Sophie, ô, Sophie ! est-ce vous que mon cœur aime ? Il l'observe, il la contemple avec une sorte de crainte et de défiance » (1926: 18 ; cité par Rosier $1999: 266)$. 
24 On songe ici au jugement de Balzac : «[...] il est négligé, incorrect à la manière des écrivains du $\mathrm{XVII}^{\mathrm{e}}$ siècle » (Honoré de Balzac, «Études sur M. Beyle » [1840], Écrits sur le roman, éd. Stéphane Vachon, Le Livre de poche, coll. «Références », 2000, p. 267).

25 Sur cette nécessité de constituer les faits de langue en faisceaux, susceptibles de définir des « appareils formels », voir Philippe 2009b : 333. Sur la pratique historique discontinuiste - foucaldienne, en somme - qui se trouve alors impliquée, voir Philippe à paraître.

${ }^{26}$ Signalons qu'il n'est pas abordé dans la thèse que Laure Lassagne a récemment consacrée aux monologues intérieurs stendhaliens (2007), et n'est pas davantage traité dans Berthier et Bordas 2005. 